\title{
Erratum to: Transdermal power transfer for recharging implanted drug delivery devices via the refill port
}

\author{
Allan T. Evans • Srinivas Chiravuri • \\ Yogesh B. Gianchandani
}

Published online: 9 February 2010

(C) Springer Science+Business Media, LLC 2010

\section{Erratum to: Biomed Microdevices \\ DOI 10.1007/s10544-009-9371-z}

The original version of this article unfortunately contained a mistake. Editing notes were inserted into the manuscript. The first paragraph of section 2, should now read:

The most important aspects of the design are power handling capability, isolation of the drug and tissue from electrical current, minimal tissue and drug heating, and ease of alignment between the needle and the port. In order to transfer DC power, the needle should be composed of at least two conductors, or poles, which must mate with corresponding poles in the refill port. The conductive path should also be electrically isolated. (The isolation is particularly important if the needle is being used to refill the drug reservoir at the same time the battery is being recharged. While this capability is not fundamentally required, it can improve efficiency and convenience.) Structural options for providing multiple conductive paths in a single needle include the use of multiple conductors within the lumen, the use of concentric isolated conductors, or splitting the needle longitudinally and isolating the halves. The split needle allows for simple alignment because it provides access to both conductors on the exterior of the needle. Sub-section 2.1 outlines the power transfer design constraints of the needle; sub-section 2.2 outlines the design of the mating mechanism and the port for use with the multi-pole needle; sub-section 2.3 describes the assembly procedure for the needle and the refill port.

Additionally, the caption for Figure 3 should now read:

Fig. 3 The septum is fabricated from biologically compatible materials. Contact springs at various heights connect to the openings in the needle insulation to allow power transfer.

The online version of the original article can be found at http://dx.doi.org/ 10.1007/s10544-009-9371-z.

\footnotetext{
A. T. Evans $(\bowtie) \cdot$ Y. B. Gianchandani

Department of Electrical Engineering and Computer Science,

University of Michigan,

1301 Beal Ave.,

Ann Arbor, MI 48109, USA

e-mail: evansall@umich.edu

Y. B. Gianchandani

e-mail: yogesh@umich.edu

S. Chiravuri

Department of Anesthesiology,

1500 East Medical Center Drive,

Ann Arbor, MI 48109-5048, USA
} 